Article

\title{
An Improved Collaborative Algorithm with Artificial Neural Network in Multidisciplinary Design Optimization of AUV
}

\author{
Xiaodong $\mathrm{Xu}{ }^{1, *}$, Honkai $\mathrm{Li}^{2}$ \\ 1 Research Institute of UAV, Nanjing University of Aeronautics and Astronautics; xdxu@nuaa.edu.cn \\ 2 College of Astronautics, Nanjing University of Aeronautics and Astronautics; hk_lee@nuaa.edu.cn \\ * Correspondence: xdxu@nuaa.edu.cn; Tel.: +86-153-6617-3956
}

\begin{abstract}
Multidisciplinary Design Optimization (MDO) is the most active field in the design of current complex system engineering, which is possessed with such two difficulties as subsystem information exchange and analytical and computational complexity of systems. Therefore, an improved collaborative optimization algorithm based on ANN (artificial neural network) response surface was proposed dependent on the consistency constraint algorithm and concurrent subspace algorithm. As an optimization method with secondary structure, it satisfied only local constraints in discipline layer, but provided a coordinated mechanism for interdisciplinary conflict in system layer. Finally, it was applied in the multidisciplinary design optimization of autonomous underwater vehicle (AUV). As shown from the result, the MDO convergence stability and reliability of low resistance, low noise and high maneuvering performance of the AUV shape can be ensured by the improved collaborative optimization algorithm, thus verifying the effectiveness of the algorithm.
\end{abstract}

Keywords: collaborative optimization algorithm; artificial neural network (ANN); low noise; low resistance; maneuvering performance

\section{Introduction}

Over the years, Autonomous Underwater Vehicles (AUVs) have a very significant value in military applications, homeland security, hydrographic surveys, mineral field surveys, air crash investigations and scientific research [1]. They are capable of carrying a variety of sensor payloads for marine geoscience research in complex ocean environment. Many efforts have been made to meet the intensive challenges of oceanographic exploration and exploitation programs.

Recently the development of AUVs is focused on improving the operation range and endurance for long term data collection in the fields of oceanography and coastal management [2]. The vehicle should be optimized with lower resistance in shape during the period of missions so that it can cruise longer distances for a long time. Therefore, hull resistance reduction is one of the key design targets in the preliminary stage of concept design [3,4].Meanwhile, maneuverability and low noise are also critical to the survey accuracy and security of AUVs [5,6]. Therefore, more attention should be paid to the multi-disciplinary optimization in form design of AUVs to minimize drag, reduce noise and improve maneuvering performance.

Multidisciplinary design optimization (MDO) techniques have been applied successfully in the scope of complex systems such as aerospace, aircraft and undersea vehicles [7,8]. It is developed on the basis of computer technology, and structural analysis and optimization technology, etc. Besides maintaining independent design optimization in discipline layer, multidisciplinary design 
technique also offers a system mechanism to coordinate the interdisciplinary conflict and get the best overall solution of the system. It carries out concurrent optimization design by means of distributed computer network environment, which can shorten the design cycle and reduce costs greatly.

With the development of MDO, optimization frameworks such as multidisciplinary feasible directions (MDF), concurrent subspace optimization (CSSO) algorithm, collaborative optimization (CO) algorithm and bi-level integrated system synthesis (BLISS) were emerged. Among them, the $\mathrm{CO}$ algorithm proposed by Kroo et al. [8] has been considered the most promising algorithm in multidisciplinary optimizations due to its good disciplinary autonomy and concurrent processing capabilities.

In traditional CO algorithm, excessive constraints of system level, increased number of computing iterations and unable convergence of optimization results are caused by the unique expression of system-level consistent restraint $[9,10]$ In this paper, an improved collaborative optimization algorithm (ICOA) which applied ANN as a response surface was proposed to solve the approximate process function, which enabled concurrent design and optimization of various disciplines to be realized. Its advantages can be presented in the following aspects: 1) accumulate disciplinary information in the design process; 2 ) find a better result in the feasible region of each iteration calculation; 3) have higher efficiency of the algorithm; 4) allow variables of the system level to be discrete variables and do not require sensitivity analysis.

On the basis of the improved multidisciplinary CO (IMCO) framework, the integrated MDO problem of low resistance, low noise and high maneuvering performance of AUV shape is decomposed into: a system level and three subsystem levels of hydrodynamic discipline, flow noise discipline and maneuverability discipline. The optimization target of hydrodynamic discipline is to reduce the submersible resistance, flow noise discipline is to reduce the AUV flow noise, and maneuverability discipline is to improve submersible maneuverability. As shown from the result, the MDO convergence stability and reliability of low resistance, low noise and high maneuvering performance of the AUV shape can be ensured by the improved CO algorithm, thus verifying the effectiveness of the algorithm.

\section{Improved CO Algorithm}

In the optimization design of actual system engineering, the following three main problems [11-13] are caused by the mathematical expression of traditional $\mathrm{CO}$ algorithm:

1) If Jacobian matrix is a singular matrix at the optimal solution, Kuhn-Tucker conditions will be unable to be satisfied, thus seriously affecting the convergence result and efficiency in system level.

2) Encountered with actually complex MDO problems, the number of subsystems is normally more than that of system-level shared variables, which causes the number of subsystem-level constraints to be larger than that of system-level. Therefore, the degree of freedom of system-level optimization is greatly limited.

3) In the actual engineering design, magnitude difference of various design variables and requirements for design accuracy are not considered in the equality constraints of system-level consistency. Lost consistency control effect of small-magnitude variables might be caused in the 
consistency constraint expressions of multi-disciplines. And the unable convergence is easily occurred in the optimization process due to the over harsh equality constraints.

As a novel modeling tool, ANN is able to simulate complex relationship between inputs and outputs in the actual system only by obtaining enough training samples, which is possessed with strong nonlinear modeling capability. Therefore, ANN is used as a response surface here to improve the traditional $\mathrm{CO}$ algorithm.

Artificial Neural Networks (ANN) is a complex concurrent network architecture formed by the connection of numerous simple processing units. Although only simple computing function is performed by each unit, the highly complex nonlinear system can be constructed by the entire network. And the curve fitting [14] closest to the reality can be acquired from the data analysis and processing by ANN. As demonstrated from Kolmogrov's Mapping Neutral Network Existence Theorem, supposing there are $\mathrm{n}$ input variables and $\mathrm{m}$ output variable in a function, a three-layer feed-forward BP neural network with $n$ neurons in the input layer, $2 n+1$ neurons in the hidden layer and $\mathrm{m}$ neurons in the output layer can be established. And in the three-layer feed-forward neural network, any continuous function can be approximated if the nonlinear increasing function is applied in the hidden layer and linear function is employed in the input and output layers, whose network topology is shown as Fig.1. The weight in the figure is the parameter to be estimated, namely the desired output. The optimal weight of neural network is obtained through sample learning, which applies BP algorithm of error back propagation to find the weight with minimal error between the actual output and expected output of samples. In this way, the original function can be approximated globally through adjusting unknown coefficients by training the neural network. S-type function is applied in the hidden layer, and the fitting function of the response surface is as follows:

$\bar{f}(x)=\sum_{k=1}^{m} w_{j k} \frac{1}{1+e^{-\sum_{i=1}^{n} x_{i} w_{i j}}}$

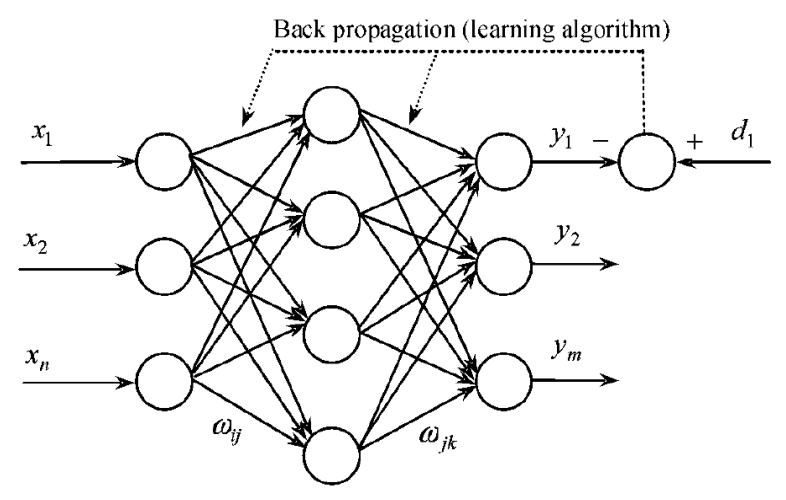

Fig.1. Topology of three-layer neural network

ANN is applied as the response surface, and then the improved system-level optimization model is:

$\operatorname{Min}: f_{s y s}$

s.t. $\quad g_{j}=\max \left(\left|x_{11}-z_{1 j}\right|,\left|x_{21}-z_{1 j}\right|,\left|x_{n 1}-z_{1 j}\right|\right) \leq \varepsilon_{j}$ 
Wherein, $j$ is the number of system-level design variables and $\varepsilon_{j}$ is the accuracy required by the $j$ th shared variable in the actual engineering.

\section{MDO Model of AUV Shape}

On the basis of the improved multidisciplinary CO framework, the integrated MDO problem of low resistance, low noise and high maneuvering performance of AUV shape is decomposed into: a system level and three subsystem levels of hydrodynamic discipline, flow noise discipline and maneuverability discipline. The optimization target of hydrodynamic discipline is to reduce the submersible resistance, flow noise discipline is to reduce the AUV flow noise, and maneuverability discipline is to improve AUV maneuverability.

The applied AUV herein is a linear structure designed by the profile expression of streamline rotator (Fig.2). Linear geometric parameters of the AUV are: total length $L=6 m$, length of the head curve segment $L_{M}=1 \mathrm{~m}$, length of the cylindrical segment $L_{H}=3.5 \mathrm{~m}$, total length of the tail $L_{T}=1.5 \mathrm{~m}$, and maximum diameter $D_{0}=0.5334 \mathrm{~m}$. The two-parameter elliptical line is applied in the curve segments of both the head and tail. The cruciform symmetrical layout is applied in fin and rudder, and the planar shapes of a single fin and rudder were shown as Fig.3: wherein, $a_{f}$ and $a_{r}$ represent the elongation of fin and rudder, respectively, $b_{f}$ and $b_{r}$ indicate the chord length of fin and rudder, respectively. The physical linear equation of the head curve segment is:

$y=\frac{D_{0}}{2}\left[1-\left(1-\frac{X}{L_{H}}\right)^{n_{h}}\right]^{1 / \mathrm{m}_{h}}$

And the physical linear equation of the tail curve segment is:

$y=\frac{D_{0}}{2}\left[1-\left(X-L_{H}-L_{M}\right)^{n_{t}}\right]^{m_{t}}$

Wherein, $m_{h}$ and $n_{h}$ are two parameters of elliptical line of the head, while $m_{t}$ and $n_{t}$ are two parameters of elliptical line of the tail, whose value range is: $2 \leq m_{h} 、 n_{h}, m_{h}, n_{h} \leq 5$

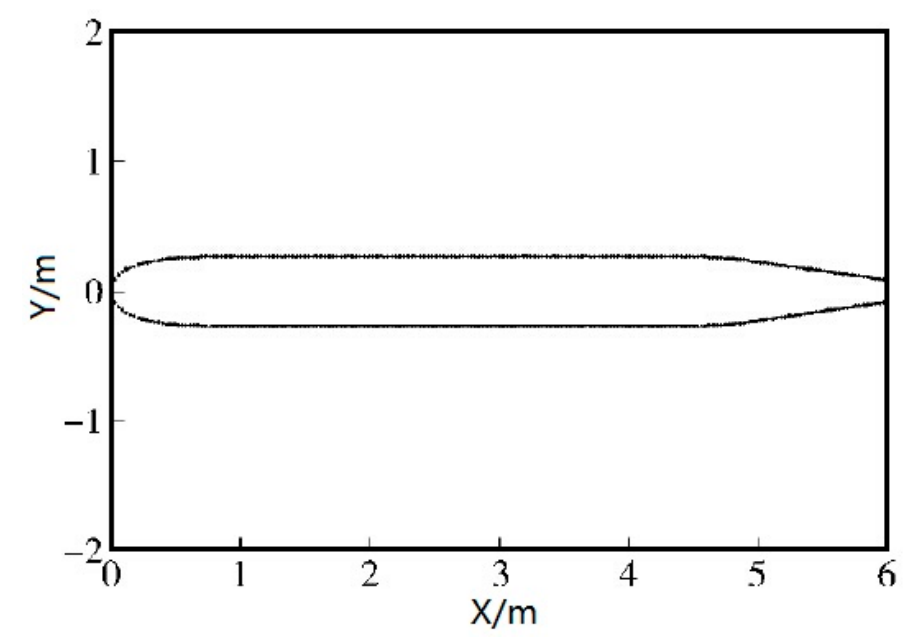

Fig.2. Streamline rotator 


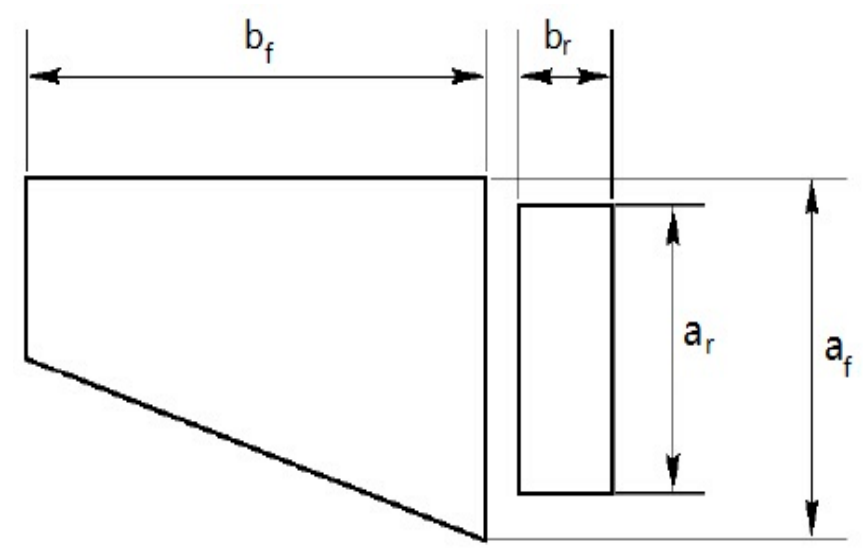

Fig.3. Diagram of planar shape of fin and rudder

\subsection{Optimization model of hydrodynamic discipline}

$$
\begin{aligned}
& \text { Min } F_{A}\left(\mathrm{X}_{A}, \overline{\mathrm{P}}_{\mathrm{PF}}, \overline{\mathrm{P}}_{B L}\right)=C_{x}\left(\mathrm{~m}_{h}, \mathrm{n}_{h}, \mathrm{~m}_{t}, \mathrm{n}_{t}, \overline{\mathrm{P}}_{\mathrm{PF}}, \overline{\mathrm{P}}_{B L}\right) \\
& \text { s.t. } G_{1 A}=\psi_{H}\left(\mathrm{~m}_{h}, \mathrm{n}_{h}\right) \geq 0.8 \\
& \qquad G_{2 A}=\alpha\left(\mathrm{m}_{t}, \mathrm{n}_{t}\right) \leq 12^{\circ} \\
& G_{3 A}=D_{E}\left(\mathrm{~m}_{t}, \mathrm{n}_{t}\right) \geq 0.1(\mathrm{~m})
\end{aligned}
$$

Wherein, $\mathrm{X}_{A}$ is the vector design of hydrodynamic discipline, $F_{A}$ refers to the optimized objective function of hydrodynamic discipline, $G_{i A}(i=1,2,3)$ indicates the constraints of hydrodynamic discipline, $\overline{\mathrm{P}}_{\mathrm{PF}}$ is the vector in the coupling state of external potential flow field and $\overline{\mathrm{P}}_{B L}$ means the vector in the coupling state of boundary layer. If resistance coefficient $C_{x}$ of zerolift adhesional wetting is the optimization objective function, its calculation formula is shown as follows:

$C_{x}=\frac{2 \pi}{\Omega} \int_{0}^{L} C_{p} \sin (\gamma) \mathrm{YdX}+\frac{2 \pi}{\Omega} \int_{0}^{L} C_{f} \cos (\gamma) \mathrm{YdX}$

Wherein, $C_{p}$ represents the surface pressure coefficient, $C_{f}$ means the friction shear stress coefficient of the surface, $\gamma$ indicates the angle between the tangent of AUV line and axis $X$, and $\Omega$ expresses the surface area of the AUV adhesion wetting. Among them, panel method is applied to solve the circling potential flow field, and finite difference method is employed to solve the boundary layer, so as to conduct the iterative calculation for equations between potential flow and boundary layer. Hence, the distributions of surface pressure and surface friction shear stress are obtained.

\subsection{Optimization model of flow noise discipline}

$\operatorname{Min} F_{B}\left(\mathrm{X}_{B}, \overline{\mathrm{P}}_{\mathrm{PF}}, \overline{\mathrm{P}}_{B L}\right)=\operatorname{SPECG}\left(\mathrm{m}_{h}, \mathrm{n}_{h}, \overline{\mathrm{P}}_{\mathrm{PF}}, \overline{\mathrm{P}}_{B L}\right)$

s.t. $G_{1 B}=\psi_{H}\left(\mathrm{~m}_{h}, \mathrm{n}_{h}\right) \geq 0.8$

Wherein, $\mathrm{X}_{B}$ is the design vector of flow noise discipline; $F_{B}$ indicates the optimized objective function of flow noise discipline; and $G_{i B}(i=1)$ means the constraints of flow noise discipline. The flow noise level radiated from the transition zone of AUV boundary layer to the stagnation point of the head is taken as the optimized objective function. Based on the Liepmann theory[15], namely 
monopole sound source model pulsated by displacement thickness, the spectral density equation of the radiated power can be shown as follows in the transition zone of boundary layer sound:

$$
\begin{aligned}
& \text { SPECG }=G_{\text {monopole }}(r, \omega) \approx \frac{W \rho^{2}\left(\Delta \delta^{*}\right)^{2} u_{0} u_{c}^{2}}{8 \pi^{2} r^{2}} \times F^{*}\left(k_{c} \Delta x, a^{*} \Delta x, \frac{u_{c} t_{i}}{\Delta x}, \frac{u_{c}}{u_{0}}\right) \\
& F^{*}\left(k_{c} \Delta x, a^{*} \Delta x, \frac{u_{c} t_{i}}{\Delta x}, \frac{u_{c}}{u_{0}}\right)=\frac{\left(k_{c} \Delta x\right)^{2}}{\left[1+\left(k_{c} \Delta x\right)^{2}\left(\frac{u_{c} t_{i}}{\Delta x}\right)^{2}\right]} \times F\left(k_{c} \Delta x, a^{*} \Delta x, \frac{u_{c}}{u_{0}}\right)
\end{aligned}
$$

Wherein, $\Delta \delta^{*}$ represents the variation of displacement thickness from laminar boundary layer to turbulent boundary layer, and $u_{c} t_{i} / \Delta x$ expresses dimensionless rise time.

\subsection{Optimization model of maneuverability discipline}

$$
\begin{gathered}
\text { Min } F_{C}\left(\mathrm{X}_{C}, \overline{\mathrm{P}}_{\mathrm{PF}}, \overline{\mathrm{P}}_{B L}\right)=R_{z \min }\left(a_{f}, a_{r}, b_{f}, b_{r}, \overline{\mathrm{P}}_{\mathrm{PF}}, \overline{\mathrm{P}}_{B L}\right) \\
\text { s.t. } 0<G_{1 C}=G_{y}\left(a_{f}, a_{r}, b_{f}, b_{r}, \overline{\mathrm{P}}_{\mathrm{PF}}, \overline{\mathrm{P}}_{B L}\right)<1 \\
\quad 0<G_{2 C}=G_{z}\left(a_{f}, a_{r}, b_{f}, b_{r}, \overline{\mathrm{P}}_{\mathrm{PF}}, \overline{\mathrm{P}}_{B L}\right)<1
\end{gathered}
$$

Wherein, $\mathrm{X}_{C}$ is the design vector of maneuverability discipline; $F_{C}$ indicates the optimized objective function of maneuverability discipline; and $G_{i C}(i=1,2)$ means the constraints of maneuverability discipline. In the horizontal plane, the least radius of gyration $R_{z \min }$ of AUV that makes rotary movement is taken as the optimized objective function, whose formula is as follows[16]:

$$
\frac{L}{R_{z \min }}=\frac{\left(C_{z}^{\beta}+C_{x s}\right) m_{y}^{\beta}+C_{z}^{\delta} m_{y}^{\beta}}{\left(C_{z}^{\beta}+C_{x s}\right) m_{y}^{\omega}-\left(\mu-C_{z}^{\omega}\right) m_{y}^{\beta}} \delta_{v \max }
$$

Wherein: $\delta_{v \max }$ is the maximum rudder angle of the vertical rudder; and $C_{x s}$ represents the zerolift resistance coefficient of the largest cross sectional area (CSA) of AUV.

\subsection{System-level optimization model}

$$
\begin{aligned}
& \operatorname{Min} F_{S}\left(\mathrm{X}_{S}\right)=\frac{\left|\bar{F}_{A}\left(\mathrm{X}_{S}\right)-F_{A}\left(\mathrm{X}_{A}^{*}\right)\right|}{F_{A}\left(\mathrm{X}_{A}^{*}\right)}+\frac{\left|\bar{F}_{B}\left(\mathrm{X}_{S}\right)-F_{B}\left(\mathrm{X}_{B}^{*}\right)\right|}{F_{B}\left(\mathrm{X}_{B}^{*}\right)}+\frac{\left|\bar{F}_{C}\left(\mathrm{X}_{S}\right)-F_{C}\left(\mathrm{X}_{A}^{*}\right)\right|}{F_{C}\left(\mathrm{X}_{C}^{*}\right)} \\
& \text { s.t. } G_{1 S}=\psi_{H}\left(\mathrm{~m}_{h}, \mathrm{n}_{h}\right) \geq 0.8 \\
& \qquad \begin{aligned}
G_{2 S} & =\alpha\left(\mathrm{m}_{t}, \mathrm{n}_{t}\right) \leq 12^{0} \\
G_{3 S} & =D_{E}\left(\mathrm{~m}_{t}, \mathrm{n}_{t}\right) \geq 0.1(\mathrm{~m}) \\
0<G_{4 S} & =G_{y}\left(a_{f}, a_{r}, b_{f}, b_{r}\right)<1 \\
0<G_{5 S} & =G_{z}\left(a_{f}, a_{r}, b_{f}, b_{r}\right)<1
\end{aligned}
\end{aligned}
$$

Wherein, $\mathrm{X}_{S}$ is the system-level design vector; $F_{S}$ indicates the optimized system-level objective function; and $G_{i S}(i=1,2,3,4,5)$ means the system-level constraints. $\mathrm{X}_{A}^{*}, \mathrm{X}_{B}^{*}$ and $\mathrm{X}_{C}^{*}$ indicate the optimized optimal solutions of hydrodynamic discipline, flow noise discipline and maneuverability discipline, respectively. $\bar{F}_{A}, \bar{F}_{B}$ and $\bar{F}_{C}$ represent the response surface agent models regarding optimized objective functions of hydrodynamic discipline, flow noise discipline and maneuverability discipline, respectively. 


\section{Multidisciplinary Optimization Result of AUV Shape}

4.1 Response surface agent model of Subsystem-level objective functions

1) ANN is applied to establish the response surface model $\bar{F}_{A}$ of the objective function of hydrodynamic discipline:

Let $x_{1}=m_{h}, x_{2}=n_{h}, x_{3}=m_{t}, x_{4}=n_{t}$, then:

$$
\begin{aligned}
& \bar{F}_{A}=\beta_{0}+\beta_{1} x_{1}+\beta_{2} x_{2}+\beta_{3} x_{3}+\beta_{4} x_{4}+\beta_{11} x_{1} x_{1} \\
& \quad+\beta_{22} x_{2} x_{2}+\beta_{33} x_{3} x_{3}+\beta_{44} x_{4} x_{4}+\beta_{12} x_{1} x_{2} \\
& \quad+\beta_{13} x_{1} x_{3}+\beta_{14} x_{1} x_{4}+\beta_{23} x_{2} x_{3}+\beta_{24} x_{2} x_{4}+\beta_{34} x_{3} x_{4}
\end{aligned}
$$

Linear equations are solved by the Gauss out PCA elimination method. It is obtained that the undetermined coefficient in the response surface model is as follows:

$$
\begin{aligned}
& \beta_{0}=-0.0073496, \beta_{1}=0.00045731 \\
& \beta_{2}=0.0016864, \beta_{3}=0.00143352 \\
& \beta_{4}=0.0015908, \beta_{11}=1.39058 \times 10^{-6} \\
& \beta_{22}=-2.7649 \times 10^{-5}, \beta_{33}=-5.6105 \times 10^{-5} \\
& \beta_{44}=-1.5014 \times 10^{-5}, \beta_{12}=-5.0092 \times 10^{-5} \\
& \beta_{13}=-3.4226 \times 10^{-5}, \beta_{14}=-2.9776 \times 10^{-5} \\
& \beta_{23}=-0.0001, \beta_{24}=-0.00023, \beta_{34}=-0.0001
\end{aligned}
$$

2) ANN is applied to establish the response surface model $\bar{F}_{B}$ of the objective function of flow noise discipline:

Let $x_{1}=m_{h}$ and $x_{2}=n_{h}$, then:

$$
\bar{F}_{B}=\beta_{0}+\beta_{1} x_{1}+\beta_{2} x_{2}+\beta_{11} x_{1} x_{1}+\beta_{22} x_{2} x_{2}+\beta_{12} x_{1} x_{2}
$$

Linear equations are solved by the Gauss out PCA elimination method. It is obtained that the undetermined coefficient in the response surface model is as follows:

$\beta_{0}=-2263.77, \beta_{1}=605.448$

$\beta_{2}=706.75, \beta_{11}=-36.2638$

$\beta_{22}=-53.1086, \beta_{12}=-92.1491$

3) ANN is applied to establish the response surface model $\bar{F}_{C}$ of the objective function of maneuverability discipline:

$$
\begin{aligned}
& \quad \text { Let } x_{1}=m_{h}, x_{2}=n_{h}, x_{3}=m_{t}, x_{4}=n_{t}, \text { then: } \\
& \bar{F}_{C}=\beta_{0}+\beta_{1} x_{1}+\beta_{2} x_{2}+\beta_{3} x_{3}+\beta_{4} x_{4}+\beta_{11} x_{1} x_{1} \\
& +\beta_{22} x_{2} x_{2}+\beta_{33} x_{3} x_{3}+\beta_{44} x_{4} x_{4}+\beta_{12} x_{1} x_{2} \\
& +\beta_{13} x_{1} x_{3}+\beta_{14} x_{1} x_{4}+\beta_{23} x_{2} x_{3}+\beta_{24} x_{2} x_{4}+\beta_{34} x_{3} x_{4}
\end{aligned}
$$

Linear equations are solved by the Gauss out PCA elimination method. It is obtained that the undetermined coefficient in the response surface model is as follows: 
$\beta_{0}=19.3052, \beta_{1}=2.3909$

$\beta_{2}=2.7131, \beta_{3}=4.9704$

$\beta_{4}=9.5031, \beta_{11}=-0.1705$

$\beta_{22}=-0.1915, \beta_{33}=-0.2541$

$\beta_{44}=-0.6939, \beta_{12}=-0.1746$

$\beta_{13}=0.0728, \beta_{14}=0.0391$

$\beta_{23}=0.0123, \beta_{24}=0.0938, \beta_{34}=-0.5417$

\subsection{Optimization solution and result analysis}

1. System-level optimization result

Global optimization result of the system-level objective function value is shown in Fig.4. It can be seen that the optimal solution is obtained after 214 steps of evolution from the initial solution, which has the optimization degree of $99.03 \%$. The global optimal solution is: $m_{h}=2.23, n_{h}=3.61$ $m_{t}=2.38, n_{t}=2.69, \psi_{H}=0.80, \alpha=5.41^{0}, D_{E}=0.31, P_{S}\left(\psi_{H}\right)=0.00091, \quad P_{S}(\alpha)=0.00225$
$P_{S}\left(D_{E}\right)=0.000204, F_{S}(X)=0.0005241, F_{A}=0.002549, F_{B}=83.77(\mathrm{~dB}), F_{C}=69.68(\mathrm{~m})$

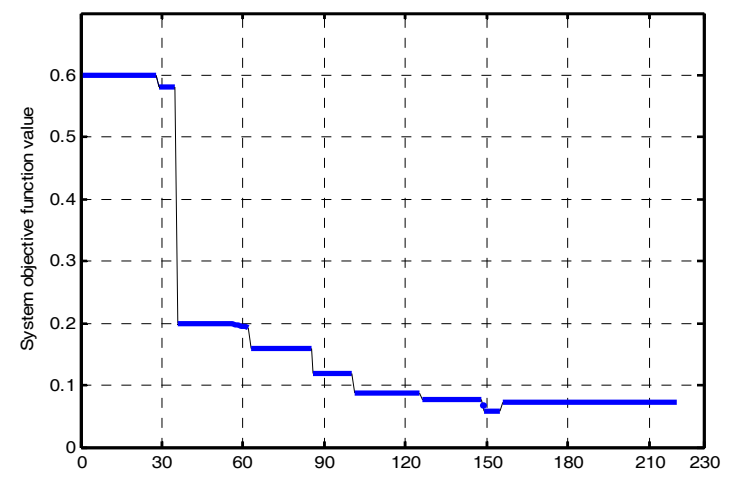

Fig.4. Optimization result of system-level objective function value

2. Optimization result of hydrodynamic discipline

Global optimization result of the objective function value of hydrodynamic discipline is shown in Fig.5. It can be seen that the optimized optimal solution of hydrodynamic discipline is $F_{A}=0.00254$. After system-level optimization, the optimal solution is $F_{A}=0.00255$, and the resistance is increased slightly with the increment of $0.029 \%$. The global optimal solution is: $m_{h}=2.94, \quad n_{h}=3.35, \quad m_{t}=2.24, \quad n_{t}=2.024, \quad \psi_{H}=0.81, \quad \alpha=7.52^{0}, \quad D_{E}=0.251$, $F_{A}=0.00214$

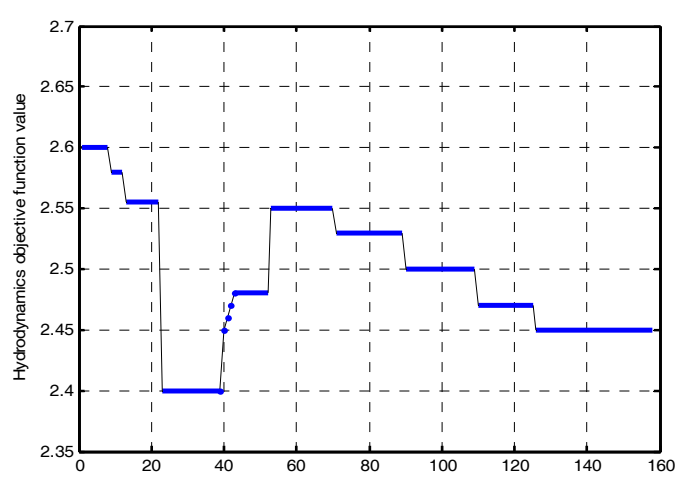

Fig.5. Optimization result of objective function value of hydrodynamic discipline 
3. Optimization result of flow noise discipline

Global optimization result of the objective function value of flow noise discipline is shown in Fig.6. It can be seen that the optimized optimal solution of flow noise discipline is $F_{B}=83.50(\mathrm{~dB})$. After system-level optimization, the optimal solution is $F_{B}=83.77(\mathrm{~dB})$, and the flow noise is reduced slightly with the reduction amount of $0.032 \%$. The global optimal solution is: $m_{h}=2.171$, $n_{h}=3.624, \quad \psi_{H}=0.805, F_{B}=83.50(\mathrm{~dB})$

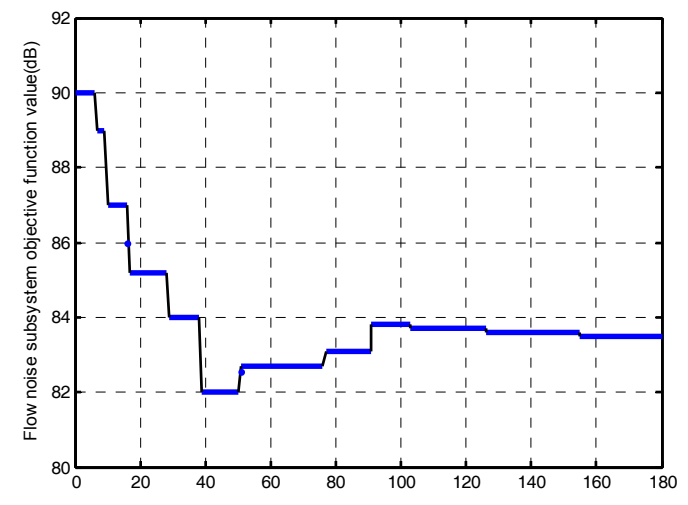

Fig.6. Optimization result of objective function value of flow noise discipline

4. Optimization result of maneuverability discipline

Global optimization result of the objective function value of maneuverability discipline is shown in Fig.7. It can be seen that the optimized optimal solution of maneuverability discipline is $F_{C}=69.50$. After system-level optimization, the optimal solution is $F_{C}=69.68$, and the least radius of gyration is enlarged slightly with the increment of $0.026 \%$. The global optimal solution is: $a_{f}=0.2677, \alpha_{r}=0.2677, b_{f}=0.271, b_{r}=0.115, G_{z}=0.794, G_{y}=0.989, F_{C}=69.47$

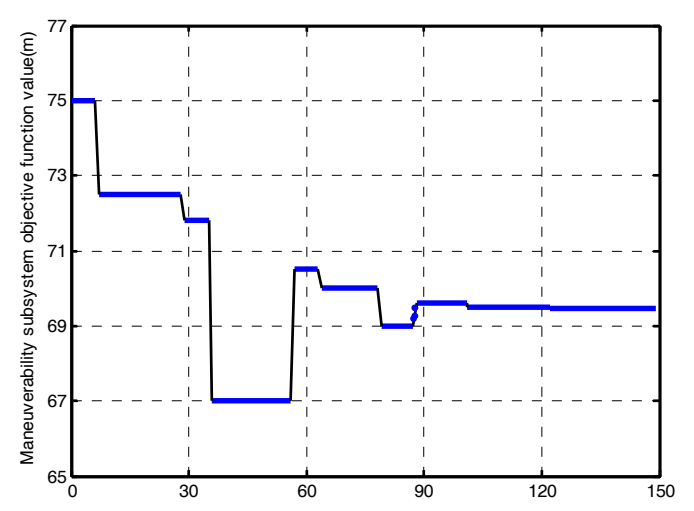

Fig.7. Optimization result of objective function value of maneuverability discipline

\section{Conclusions}

Traditional CO drawbacks appeared in solving multidisciplinary problems are analyzed in the paper. Meanwhile, an improved collaborative algorithm is proposed through the application of ANN as a response surface. On the basis of high disciplinary autonomy and concurrent design thought of conventional $\mathrm{CO}$ algorithm, it can effectively ensure the existence of feasible domain solution. A complete set of MDO methods and models integrated with low resistance, low noise and high maneuvering performance of AUV shape are established dependent on the improved CO, and ANN is applied to build the response surface model of subsystem-level objective functions. The state variable required by the system-level optimization is obtained by the response surface, which thus resolves the coupling problem between the system level and subsystem level, reduces the 
computational amount of system-level optimization and improves the efficiency of the overall optimization. As shown from the result, the ICOA method is proven to have a rapid convergence and hypothetical issues including the stability and reliability of low resistance, low noise and high maneuvering performance of the AUV shape can be ensured by the improved collaborative optimization algorithm, thus verifying the effectiveness of the algorithm.

\section{Acknowledgments:}

This work was supported by the Fundamental Research Funds for the Central Universities (NS2015097)

Author Contributions: Xiaodong Xu designed and implemented the optimization procedure proposed in this paper. Hongkai Li contributed with modeling and simulation and as well as a part of data analysis.

Conflicts of Interest: The authors declare no conflict of interest

\section{References}

1. Fernandes, P.G., Stevenson, P., Brierley, A.S., Armstrong, F., Simmonds, E.J. Autonomous underwater vehicles: future platforms for fisheries acoustics [J]. ICES Journal of Marine Science. 2003, 60, 684-691.

2. Desa E, Madhan R, Maurya P. Potential of autonomous underwater vehicles as new generation ocean data platforms[J]. Current Science, 2006, 90: 1202-1209.

3. Husaini M, Samad Z, Arshad M R. Autonomous Underwater Vehicle Propeller Simulation using Computational Fluid Dynamic [J]. Computational Fluid Dynamics Technologies and Applications, 2011: 293-314.

4. Husaini M, Samad Z, Arshad M R. CFD simulation of cooperative AUV motion[J]. Indian Journal of Geo-Marine Sciences, 2009, 38:346-351.

5. Liu Y, Fang P, Bian D, et al. Fuzzy comprehensive evaluation for the motion performance of autonomous underwater vehicles[J]. Ocean Engineering, 2014, 88: 568-577.

6. Grenon G, An P E, Smith S M, et al. Enhancement of the inertial navigation system for the Morpheus autonomous underwater vehicles[J]. Oceanic Engineering, 2001, 26: 548-560.

7. Belegundu A D, Halberg E, Yukish M A, et al. Attribute-based multidisciplinary optimization of undersea vehicles[J]. AIAA paper, 2000, 4865.

8. Kroo I, Altus S, Braun R, et al. Multidisciplinary optimization methods for aircraft preliminary design. Proc. 5th AIAA/NASA/USAF/ISSMO Symp. on Multidisciplinary Analysis and Optimization, Florida,USA,1994, Vol. 1. AIAA Paper No. 94-4325, pp. 697-707

9. Agte J, de Weck O, Sobieszczanski-Sobieski J, et al. MDO: assessment and direction for advancement-an opinion of one international group[J]. Structural and Multidisciplinary Optimization, 2010, 40: 17-33.

10. Li Hai-yan,Ma Ming-Xu, Huang Zhang-jun, et al. New adaptive penalty scheme for collaborative optimization[J].Journal of system simulation, 2009 19: 6178-6182.

11. Alexandrov N M, Lewis R M. Analytical and computational aspects of collaborative optimization for multidisciplinary design[J]. AIAA journal, 2002, 40: 301-309.

12. Zhou W, Wang D, Sheng J, et al. Collaborative optimization of maintenance and spare ordering of continuously degrading systems[J]. Systems Engineering and Electronics, 2012, 23: 63-70.

13. Fang $Y X$, Zhu B Q, Li D B. Collaborative optimization of global and local with forging multi-level and multi-objective in random environment[J]. Computer Integrated Manufacturing Systems, 2012, 18: 1182-1194.

14. Scarselli F, Chung Tsoi A. Universal approximation using feedforward neural networks: A survey of some existing methods, and some new results[J]. Neural networks, 1998, 11: 15-37.

15. Bogey C, Bailly C, Juvé D. Computation of flow noise using source terms in linearized Euler's equations[J]. AIAA journal, 2002, 40: 235-243.

16. Chiu F C, Guo J, Huang C C, et al. On the linear hydrodynamic forces and the maneuverability of an unmanned untethered submersible with streamlined body[J]. Journal of Society of Naval Architects of Japan, 1996, 180: 241-249. 